\title{
Brief Introduction of a New Kind of Glide Path Antenna
}

\author{
Chunqing Qu \\ East China Regional Air Traffic Management Bureau of Civil Aviation of China, Shanghai, China \\ Email: qcq04@163.com
}

How to cite this paper: Qu, C.Q. (2018) Brief Introduction of a New Kind of Glide Path Antenna. Open Journal of Antennas and Propagation, 6, 60-72.

https://doi.org/10.4236/ojapr.2018.63006

Received: September 10, 2018

Accepted: September 26, 2018

Published: September 29, 2018

Copyright (C) 2018 by author and Scientific Research Publishing Inc. This work is licensed under the Creative Commons Attribution International License (CC BY 4.0).

http://creativecommons.org/licenses/by/4.0/

\section{(c) (i) Open Access}

\begin{abstract}
A new kind of image type glide path beacon subsystem of Instrument Landing System (ILS) was reported. Capture effect is designed to overcome far-field (FF) reflectors and rising terrain. Low angle CSB signal was highly depressed, was $-41 \mathrm{~dB}$ at $1^{\circ}$ comparative to the max value. Both Carrier and Side Bands (CSB) and Side and Bands Only (SBO) signals were radiated by three antennas. Difference in Depth of Modulation (DDM) provided good linear variation around glide path angle $(\theta)$. At near field area, the site of near-field (NF) monitor was just about $57 \mathrm{~m}$ in front of the system, needed less length of reflection plane than that of $\mathrm{M}$-array system, result from the system had only $90 \%$ of the M-Array antenna height.
\end{abstract}

\section{Keywords}

Glide Path Antenna, Near Field Monitor Antenna, Signal Analysis, DDM

\section{Introduction}

The Instrument Landing System (ILS) had its beginnings in the United States and England during the years 1939 to 1945 [1]. Since then, ILS became the international standard system for approach and landing guidance. ILS was adopted by International Civil Aviation Organization (ICAO) in 1947 and will be in service until at least 2020 [2]. The ILS normally consists of VHF "Localizer (LOC)" for runway alignment guidance, an UHF "Glide Path (GP)" for elevation guidance and "Marker Beacons (MB)" for providing key checkpoints along the approach. The MB is replaced or supplemented, at some time, by a "Distance Measuring Equipment (DME)" to provide continuous reading of distance. The ILS, in its present $90 / 150 \mathrm{~Hz}$ format, supports lateral guidance by LOC and longitudinal guidance by GP. For GP, produces a course formed by the intersection of the antenna and its image for horizontal polarization. One pattern is mod- 
ulated by $90 \mathrm{~Hz}$ and the other by $150 \mathrm{~Hz}$. The Course Line (CL) is the vertical plane where the $90 \mathrm{~Hz}$ and $150 \mathrm{~Hz}$ modulations are equal. When the aircraft is to the left of the course in the predominately $90 \mathrm{~Hz}$ region, the signals received by the airborne receiver will produce a "fly right" indication for the pilot.

A series of glide path antennas were produced. Such as image type: null reference glide path antenna [3]. It is the simplest one, but needs extensive smooth ground plane, and is the most affected by rising terrain or reflector in the FF; band side reference glide path antenna [4]. Contributed to its lower height, this kind of GP antenna requires the least amount of smooth ground plane. However, the immediate ground plane needs more stringent smoothness criteria; $\mathrm{M}$-array glide path antenna [5]. It is also called capture effect, using two frequencies to provide course and clearance. In the above three types, this one is the best choice for difficult sites originated from the most tolerant to FF reflectors and rising terrain; and non-image type: end-fire glide path [6]. Mainly used to lateral terrain is limited location, so the end-fire does not need a plane to form image pattern.

In the paper, a new kind of image type glide path antenna system is introduced. It is similar to the M-array glide path antenna system, radiated by three antennas with capture effect. Since it uses separate transmitters to provide the course and clearance, it is tolerant to far-field reflectors and rising terrain. Moreover, this new type could tolerant to more difficult sites and needs less size of smooth ground and reflection plane. Because this system looks as a shrunk M-Array system, called S-Array glide path antenna. The discussion below introduces the structure of the S-Array as well as its signal distribution. And then, the $3 \mathrm{D}$ pattern of the space signal would be exhibited. Meanwhile the near field characteristic should be analyzed detailed. At last, the several parameter of S-Array is compared with other kind of image type glide path beacon and proved the best performance.

\section{Result and Discussion}

\subsection{Antenna Introduction}

\subsubsection{Antenna Site}

The S-Array glide path antenna system consists of three antennas, upper, middle and lower antenna. $h_{1}=1.3 h, h_{2}=2.0 h$ and $h_{3}=2.7 h$ respectively. The structure of the antenna and the position of the near field monitor antenna can be found in Figure 1 . Where $h=\frac{\lambda}{4 \sin (\theta-F S L)}, \theta$ is the glide angle, generally $\theta$ is $3^{\circ}$, and Forward Slope $(F S L)$ is forward slope in front of the antenna in first Fresnel zone. The frequency used below is $333.35 \mathrm{MHz}$ and corresponding $\lambda$ is $0.9 \mathrm{~m}$. And $h_{1}=5.6 \mathrm{~m}, h_{2}=8.6 \mathrm{~m}, h_{3}=11.6 \mathrm{~m}$. Meanwhile the near field monitor (NF) is placedat a distance $(D)$ about $57 \mathrm{~m}$ in front of the antenna system. The phase error between $h_{1}$ and $h_{3}$ to NF is $360^{\circ}$. And the height is little higher than the value $D \cdot \tan \theta$, mainly depends on tested value on the spot after the flight check. 


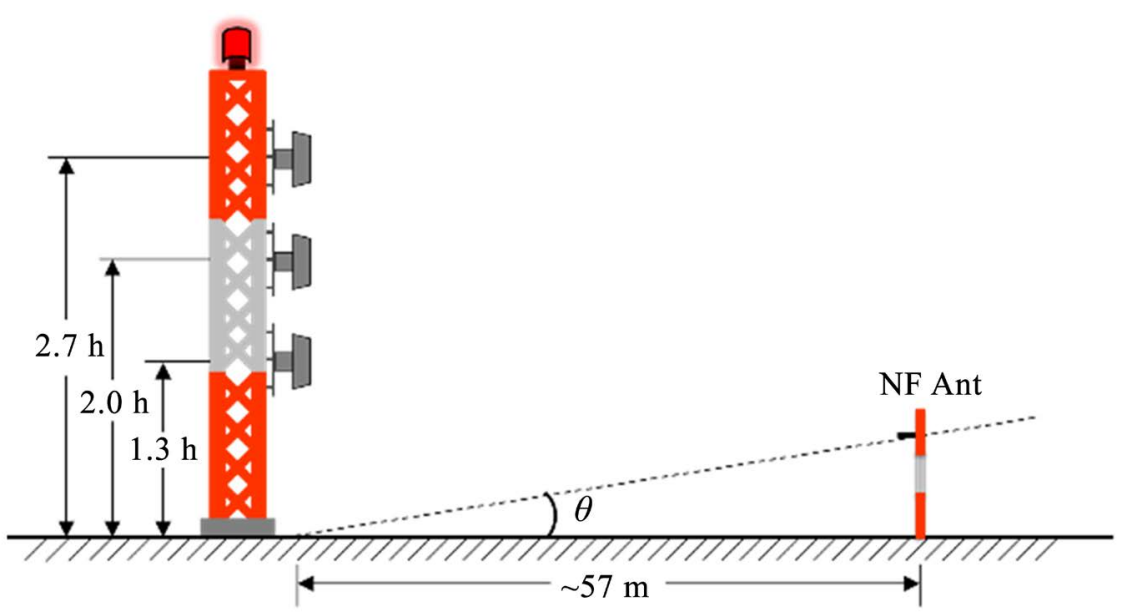

Figure 1. (Color Online) The site of the S-type glide path antenna and the near-field monitor. The system consists of three uniformly spaced antennas, upper, middle and lower antenna. And their heights are $1.3 h, 2.0 h$ and $2.7 h$, where $h$ is the height of lower antenna of null reference system. The site of near-field monitor antenna is about $57 \mathrm{~m}$ in front of the system. This position receives a reversed phase of ddm and the elevation angle, correspond to $\mathrm{ddm}=0$ point, closes to the glide path angle.

\subsubsection{Antenna System Signal Distribution}

The relative amplitude and phase for standard CSB, SBO, and Clearance (CLR) feeding of the three antennas are showed in Table 1. In antenna system distribution (ADU) the SBO amplitude is not related to CSB, the feeding coefficient $k$ is changed by adjust SBO level, and the same to that of CLR.

\subsubsection{Coverage and Lobbing Diagrams (CSB, SBO, CLR)}

The coverage range requirement can be found in Figure 2 .

The lobbing diagram of three kinds of signal: CSB, SBO and CLR listed from 0 to $2 \theta$ are showed in Figure 3.

The space pattern of kathrein antenna is showed in Figure 4. From the side view of CSB coverage, to make up for lower angle coverage, the lobe is predominant by clearance instead of course. This design avoids reflecting from the far field obstacles effectively. Similarly, the SBO signal in lower angle $(150 \mathrm{~Hz}$ predominant) own the lesser signal comparative to that of $90 \mathrm{~Hz}$.

\subsubsection{DDM Curve and Feeding Coefficient for SBO Signal: $k$}

The theoretical course and clearance radiation patterns are described in Figure 5. The ddm function expression is:

$$
\mathrm{ddm}=2 \frac{E_{\mathrm{SBO}}}{E_{\mathrm{CSB}}} \cos \left(\varphi_{\mathrm{SBO}}-\varphi_{\mathrm{CSB}}\right) .
$$

The value of $\mathrm{ddm}$ is a constant value 0.4 for clearance signal predominant, while for course signal predominant, the value is twice the quotient of vector $E_{\mathrm{SBO}}$ divided by $E_{\mathrm{CSB}}$. The boundary, the red cross-point, of them is marked in the top of the figure.

There are three requirements for $\mathrm{ddm}$ distribution around the glide angle $\theta$. 
Table 1. standard antenna distribution values of S-Array.

\begin{tabular}{ccccc}
\hline & Height & CSB & SBO & CLR \\
\hline Upper Ant & $2.7 h$ & $0.14 \angle 0^{\circ}$ & $90 \mathrm{~Hz} 0.093 \angle 0^{\circ}$ & $90 \mathrm{~Hz} 0.125 \angle 0^{\circ}$ \\
& & & $150 \mathrm{~Hz} 0.093 \angle 180^{\circ}$ & $150 \mathrm{~Hz} 0.375 \angle 0^{\circ}$ \\
Middle Ant & $2.0 h$ & $0.88 \angle 180^{\circ}$ & $90 \mathrm{~Hz} 0.173 \angle 180^{\circ}$ & NA \\
& & & $150 \mathrm{~Hz} 0.173 \angle 0^{\circ}$ & $90 \mathrm{~Hz} 0.093 \angle 0^{\circ}$ \\
Lower Ant & $1.3 h$ & $1.0 \angle 0^{\circ}$ & $150 \mathrm{~Hz} 0.093 \angle 180^{\circ}$ & $90 \mathrm{~Hz} 0.125 \angle 0^{\circ}$ \\
& & & & $150 \mathrm{~Hz} 0.375 \angle 0^{\circ}$ \\
\hline
\end{tabular}

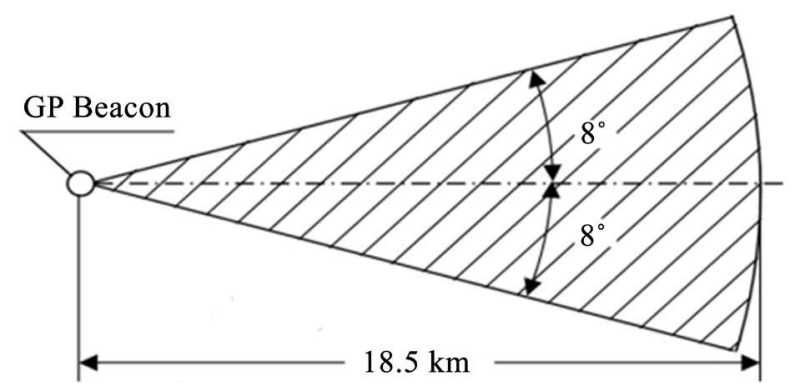

a) horizontal

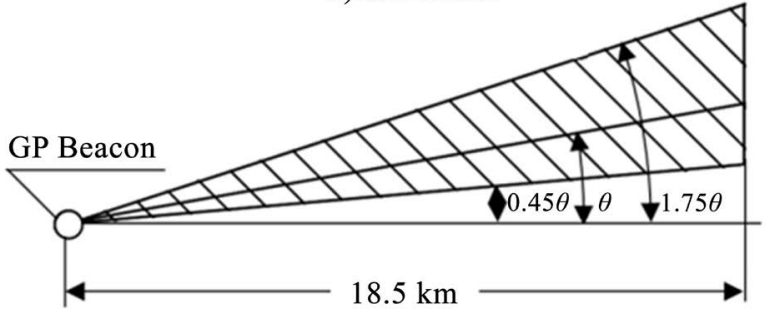

Figure 2. (Color Online) Horizontal and vertical coverage ruled by Annex 10 [7], where a distance of at least $18.5 \mathrm{~km}$ up to $1.75 \theta$ and down to $0.45 \theta$ above the horizontal, and in sectors of $8^{\circ}$ in azimuth on each of the centre line of the ILS glide path. The minimum field in this region must be more than $400 \mu \mathrm{V} / \mathrm{m}$.

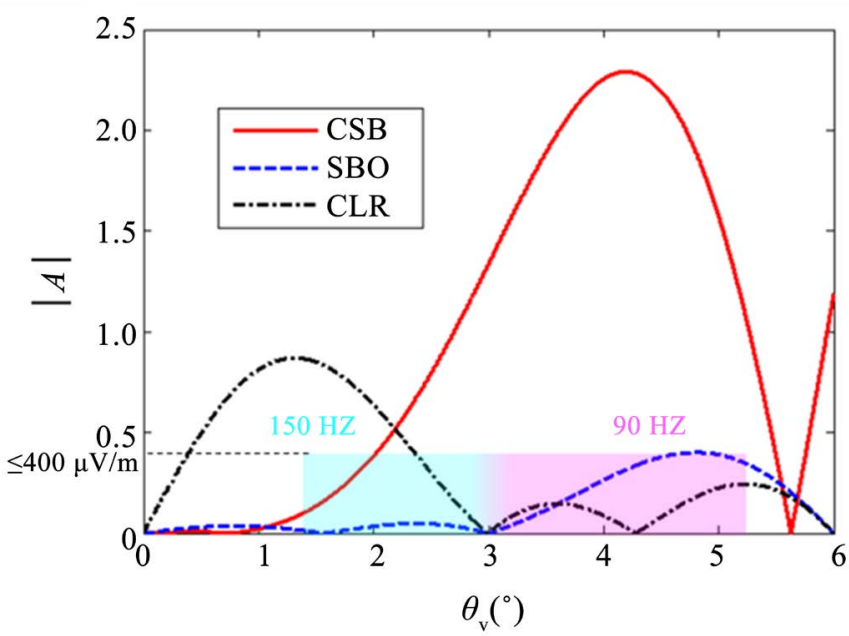

Figure 3. (Color Online) Elevation angle dependence of the absolute value of amplitude for the radiation pattern of CSB, SBO and CLR. The cyan coverage region indicates the $150 \mathrm{~Hz}$ predominant below the glide path angle $\left(1.35^{\circ}-3^{\circ}\right)$ and the pink region shows the $90 \mathrm{~Hz}$ predominant above the $\theta\left(3^{\circ}-5.25^{\circ}\right)$. 


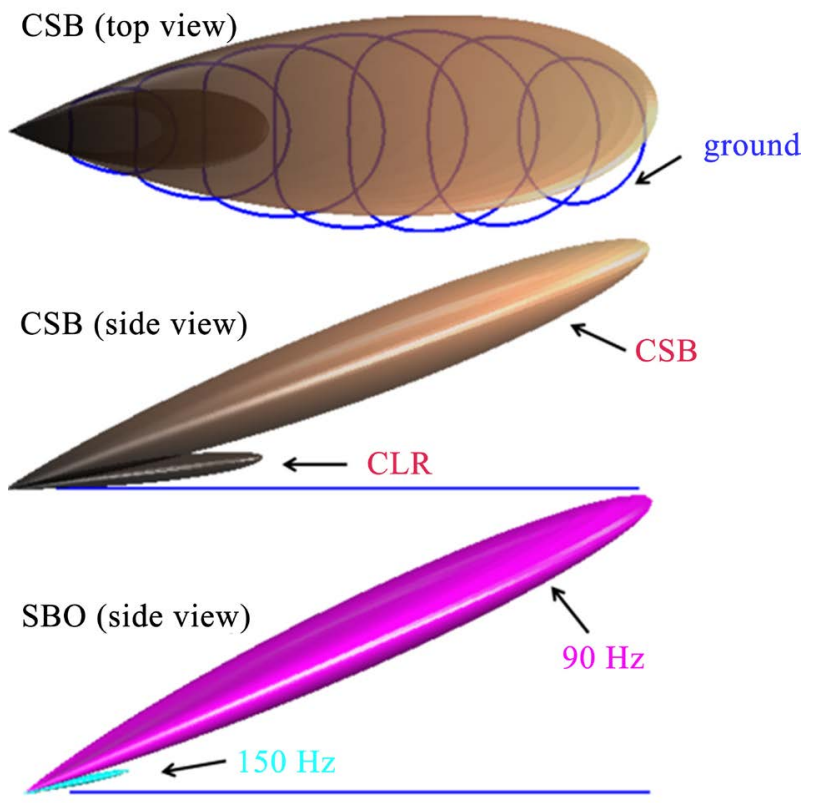

Figure 4. (Color Online) 3D pattern of glide path lobbing diagram. The range of CSB is $0^{\circ}-5.5^{\circ}$ for elevation angle and $-25^{\circ}-25^{\circ}$ for azimuth respectively. The range of SBO is $0^{\circ}-6^{\circ}$ for elevation and $-25^{\circ}-25^{\circ}$ for azimuth, which is the main lobe of Kathrein antenna for horizon [5]. The blue circle represents the ground plane. In order to have a good sight of view, the vertical angle has been enlarged five times.

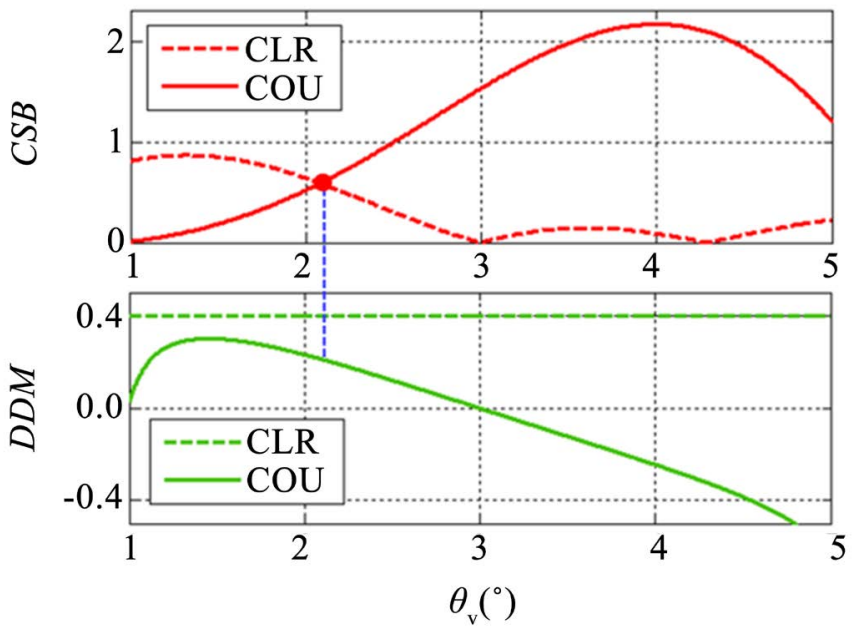

Figure 5. (Color Online) Elevation angle dependence of CSB and ddm distribution. The top diagram indicates the cross point of the absolute value of amplitude of course and clearance. This is the dividing point to decide the type of ddm. The stronger signal of CSB predominates corresponding ddm curve.

Good linearity characteristic, symmetry and $75 \mu \mathrm{A}(\mathrm{ddm}=0.0875)$ at $0.88 \theta$. To get $75 \mu \mathrm{A}, \mathrm{ddm}=0.0875$ for lower half sector at $0.88 \theta$, the coefficient $k_{1}$ for SBO relative level is:

$$
\frac{7}{160} \cdot \frac{\sin (1.3 h \sin (0.88 \theta-F S L))-0.88 \sin (2 h \sin (0.88 \theta-F S L))+0.14 \sin (2.7 h \sin (0.88 \theta-F S L))}{-0.535 \sin (1.3 h \sin (0.88 \theta-F S L))+\sin (2 h \sin (0.88 \theta-F S L))-0.535 \sin (2.7 h \sin (0.88 \theta-F S L))}
$$




$$
\begin{aligned}
& \text { where the } h \text { is electric length: } h=\frac{\pi}{2 \sin (\theta-F S L)} \\
& \qquad \mathrm{ddm}=-0.0875 \text { for upper half sector at } 1.22 \theta \text {, the coefficient } k_{2} \text { is: } \\
& \frac{-7}{160} \cdot \frac{\sin (1.3 h \sin (1.22 \theta-F S L))-0.88 \sin (2 h \sin (1.22 \theta-F S L))+0.14 \sin (2.7 h \sin (1.22 \theta-F S L))}{-0.535 \sin (1.3 h \sin (1.22 \theta-F S L))+\sin (2 h \sin (1.22 \theta-F S L))-0.535 \sin (2.7 h \sin (1.22 \theta-F S L))}
\end{aligned}
$$

Approximately, $k_{1}=k_{2}=0.173$. The further calculation results with different FSL from $-0.5^{\circ}$ to $0.5^{\circ}$ are listed in Table 2 . The data show that the coefficient $k_{1}$ is decreased with the increasing of FSL. And the error of $k_{1}$ and $k_{2}$ is negligible, which demonstrates the good symmetry. Discussion about ddm curves below would further demonstrate the linear variation around $\theta$.

Three curves of ddm distribution are exampled in Figure 6, flat ground and the ground with a forward slope for $F S L= \pm 0.5^{\circ}$. It can find that the course ddm shorted while the FSL rise, meanwhile the feeding coefficient increase. Seen in green curve, the linearity fades to be destroyed when the elevation angle surpass $4^{\circ}$. But for other two curves, both of them possess better linearity. In half glide path section region, ddm from -0.0875 to 0.0875 , all of three curves are perfectly coincident. This shows the good linear behavior and good symmetry.

\subsubsection{Alignments (Antenna Height, Lateral Offset, Longitudinal Offset)}

The antenna elements should be aligned along a straight line, it shall be perpendicular to the average forward slope $(F S L)$. If the forward slope is rising, the lower antenna shall be forward compared to the middle one.

The sideways offset of the antenna elements shall be accurately adjusted. Orientation is such that the upper antenna is closer to the runway than the middle one. And the middle antenna shall be closer to the runway than the lower one.

Detailed, for the formula below, FSL positive if ground rising toward threshold, SSL positive if ground plane rising from antenna mast toward runway.

The formula of the alignment parameters are listed below:

The longitudinal offset lower to upper $\left(s f t_{\mathrm{d}}\right)$ is: $1.4 h \cdot \sin (F S L)$, middle to upper $\left(s f t_{\mathrm{m}}\right)$ is $0.7 \mathrm{~h} \cdot \sin (F S L)$. And lateral offset upper to middle $\left(o f f_{\mathrm{u}}\right)$ is:

Table 2. SBO feeding coefficient: $k$.

\begin{tabular}{cccc}
\hline FSL $\left({ }^{\circ}\right)$ & $k_{1}(\%)$ & $k_{2}(\%)$ & $\Delta k\left(10^{-6}\right)$ \\
\hline-0.5 & 20.21 & 20.23 & -19 \\
-0.3 & 19.06 & 19.07 & -15 \\
-0.1 & 17.90 & 17.92 & -12 \\
0 & 17.33 & 17.34 & -93 \\
0.1 & 16.75 & 16.76 & -69 \\
0.3 & 15.60 & 15.60 & -14 \\
0.5 & 14.45 & 14.45 & 52 \\
\hline
\end{tabular}

$k_{1}$ for $0.88 \theta, k_{2}$ for $1.12 \theta$ and $\Delta k=k_{1}-k_{2}$. 


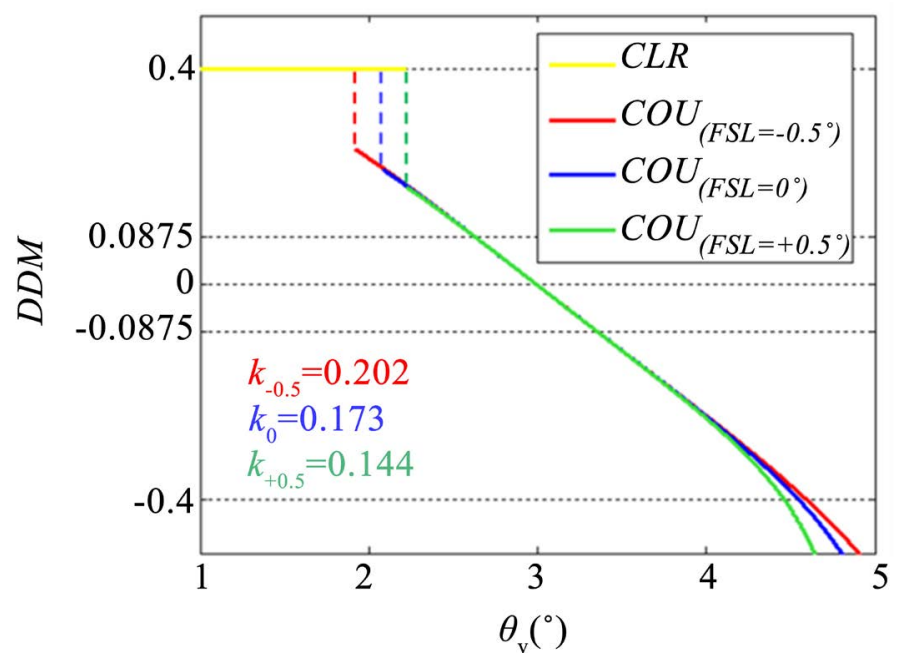

Figure 6. (Color Online) Elevation angle dependence of ddm for three circumstances: no forward slope $\left(F S L=0^{\circ}\right)$ and $F S L= \pm 0.5^{\circ}$. Below the crosspoint referred in Figure 5, the $\mathrm{ddm}$ curve transfer course to clearance with a constant value: 0.4 . The modulation of 150 $\mathrm{Hz}$ component is three time of $90 \mathrm{~Hz}$ modulation, listed in table one, so ddm $(\mathrm{CLR})=$ $\mathrm{m} 150-\mathrm{m} 90=0.6-0.2=0.4$. Three curves almost coincide within the region from $2.64^{\circ}$ $-3.36^{\circ}$ ( $\mathrm{ddm}$ from -0.0875 to 0.0875 ). Further proves the good symmetry and linear variation around $\theta$.

$$
\frac{\left(2^{2}-2.7^{2}\right) \cdot h^{2}}{2 \cdot D}+h \cdot \sin (S S L),
$$

lower to middle $\left(o f f_{\mathrm{d}}\right)$ is:

$$
\frac{\left(2^{2}-1.3^{2}\right) \cdot h^{2}}{2 \cdot D}+h \cdot \sin (S S L)
$$

where $h$ is: $h=\frac{\lambda}{4 \sin (\theta-F S L)}$.

It is worth noting that the Side Slope (SSL) just affects sft only, but $F S L$ would affect the height of antenna, off and sft.

The case of $F L S=0$ and $D=120 \mathrm{~m}: h_{1}=5.58 \mathrm{~m}, h_{2}=8.59 \mathrm{~m}, h_{3}=11.60 \mathrm{~m}$, off $_{\mathrm{u}}$ $=-25.3 \mathrm{~cm}$, off $=17.7 \mathrm{~cm}$.

\subsection{Near Field Analysis}

The signal synthesis in near field (NF) region is different to that in far field. The boundary of them is the horizontal distance $(1160 \mathrm{~m})$ that 100 times of the $h_{3}$. Outside the range of this distance, the radio signals for the direct and its image one are considered as parallel. But within this distance, they are not regarded as parallel any more. The phase error appears and should be revised. The follow discussion introduces the location of NF monitor and the NF behavior for ddm distribution.

\subsubsection{Phase Error}

The phase of the total received signal at the monitor point $\mathrm{M}$ will be as shown in 
Figure 7. Where $\Phi_{M}=\left(\Phi_{r}+\Phi_{d}\right) / 2$, and $\Phi_{r}$ is the electrical distance from radiated antenna to the receive point of the direct signal, similarly $\Phi_{d}$ works as the electrical distance of the reflected signal.

To monitor the glide path angle, the phase error should be $0^{\circ}$ or $360^{\circ}$. For a nearly $0^{\circ}$ phase error, the distance must be more than one kilometer. This distance corresponds to a very high monitor antenna. But for $360^{\circ}$ phase error, the distance is approximately $57 \mathrm{~m}$. The phase error $\Phi_{M U}-\Phi_{M L}$ for $\theta=3^{\circ}$ as a function of the distance $L$ in front of the antenna system is shown in Figure 8. The former three kinds of glide path antenna, null reference (NR), sideband reference (SR) and M-Array, are also listed for comparison. The type of NR and SR are possessed of two antennas, neither $0^{\circ}$ nor $360^{\circ}$ are convenient, for a nearly $0^{\circ}$ phase error the distance must be more than $500 \mathrm{~m}$, while for $360^{\circ}$ phase error the distance is $30 \mathrm{~m}$ for NR and $21 \mathrm{~m}$ for SB. This very short distance results in a $16^{\circ}$ elevation angle for NR and $20^{\circ}$ for SR to ground reflected signal from the upper antenna element. So the monitor position chosen for $180^{\circ}$ is advisable, the

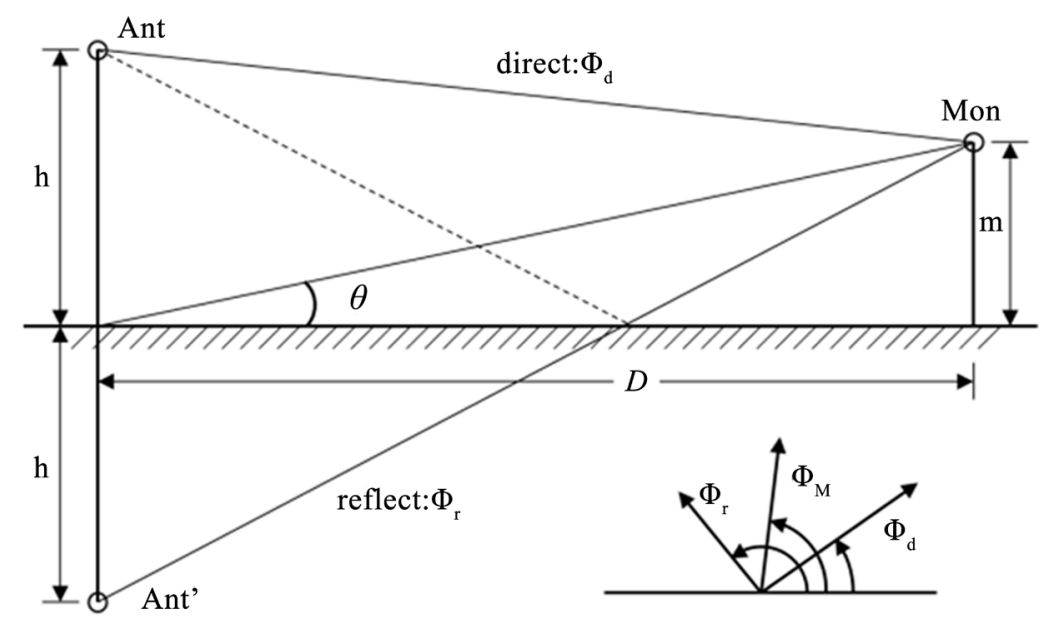

Figure 7. (Color Online) Path distance from antenna element and it's image to monitor point. The inset indicates the formation of the phase of the total signal at receive point.

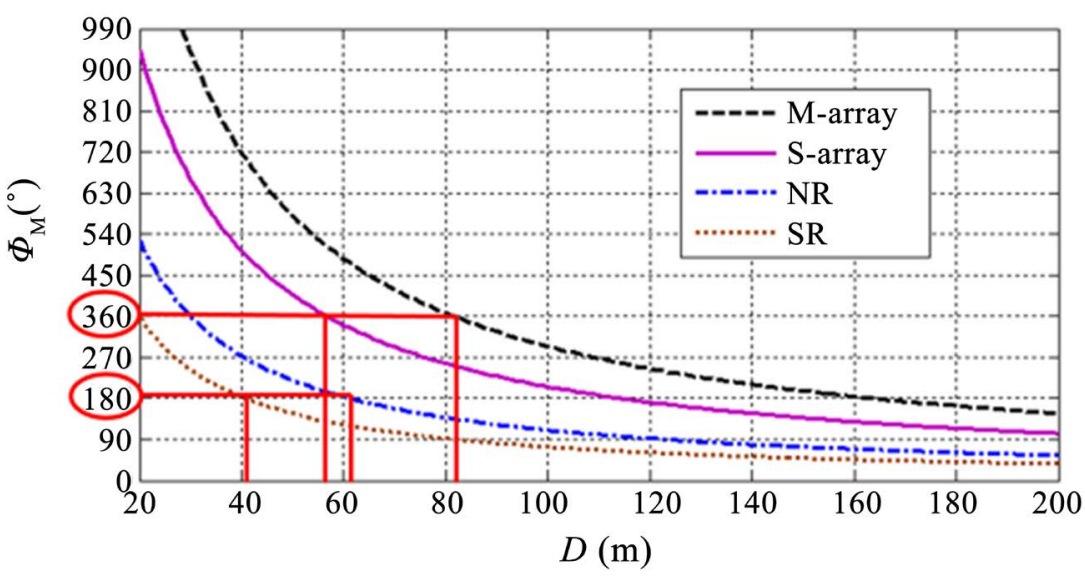

Figure 8. (Color Online) Distance dependence of NF phase error for four kinds of GP antenna. The red lines indicate the distance of their corresponding NF position. 
distance is approximately $62 \mathrm{~m}$ to capture the zero point of $\mathrm{ddm}$ for NR. In particular, the monitor antenna located at about $41 \mathrm{~m}$ and $2 \theta\left(6^{\circ}\right)$ to obtain the zero point of ddm for SR. Similar to S-Array, the distance is approximately $82 \mathrm{~m}$ for M-Array. Above all, besides the distance for SR $(\sim 41 \mathrm{~m})$, the S-Array one be possessed of a shortest distance of the currentimage type glide path antenna. It means the height of monitor antenna is also the lowest among four types. These characteristic is advantage to install and maintain, at the same time, reduce the cost.

\subsubsection{DDM Pattern}

For near field monitor, the ddm curve is essential to study. This is the monitoring basis from which the far field space signal distribution could be speculated. So the ddm distribution at $57 \mathrm{~m}\left(\Phi_{M U}-\Phi_{M L}=360^{\circ}\right)$ from the antenna system is given in Figure 9. Around the monitor point $(\mathrm{ddm}=0)$ the symmetry of the distribution becomes decayed. Specifically, the lower and upper half sections $(\mathrm{ddm}= \pm 0.0875)$ are $2.887^{\circ}$ and $3.164^{\circ}$ respectively. In fact, the ddm distribution has been disrupted. It can be found that the second glide path appears at approximate $3.6^{\circ}$. This is the false glide path, among $3^{\circ}-3.6^{\circ} 150 \mathrm{~Hz}$ predominant, besides $90 \mathrm{~Hz}$ predominant from $1.5^{\circ}$ to $4.5^{\circ}$ region.

\subsubsection{Theoretical Glide Path ( $\mathrm{NF} d \mathrm{dm}=0$ )}

Despite the alignment of lateral offset, the ddm curve would remain disrupted in $\mathrm{NF}$, especially near the threshold. The direct result is the deviation of the zero point of $\mathrm{ddm}$. In approaching range, the ddm distribution from $600 \mathrm{~m}$ to the threshold is presented in Figure 10. In order to have a good vision, the $\mathrm{ddm}$ is the absolute value, and the write line at bottom represents the line of $\mathrm{ddm}=0$. Clearly, with the distance closes to threshold, the ddm curve moves the upper angle, also the zero point of $\mathrm{ddm}$ follows to the high elevation angle. Finally, this leads to the glide path upturned.

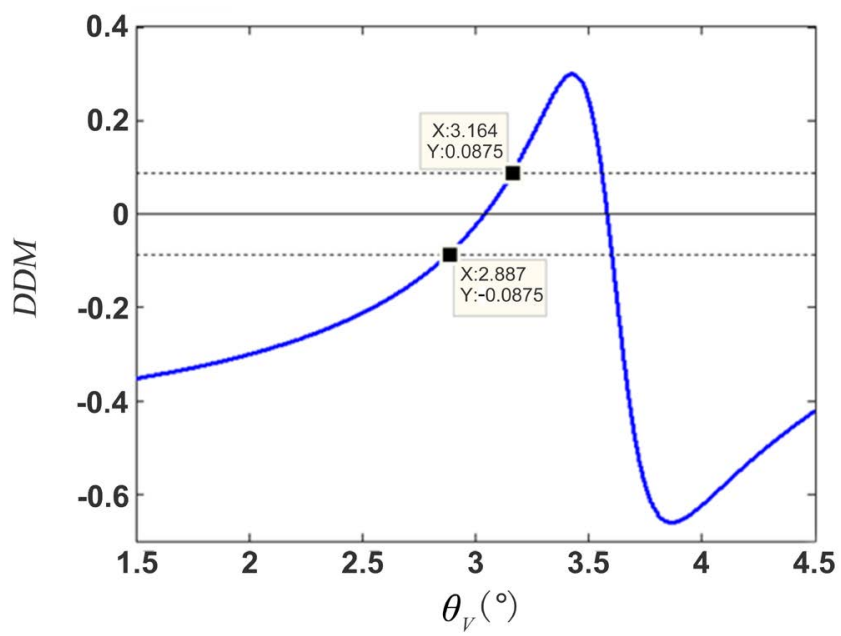

Figure 9. (Color Online) Elevation angle dependence of ddm of NF with distance is $57 \mathrm{~m}$ for S-Array. The datatip indicates elevation angle values corresponding $\mathrm{ddm}$ is \pm 0.0875 , $2.887^{\circ}$ and $3.164^{\circ}$ respectively. This shows the symmetry has become decayed. 


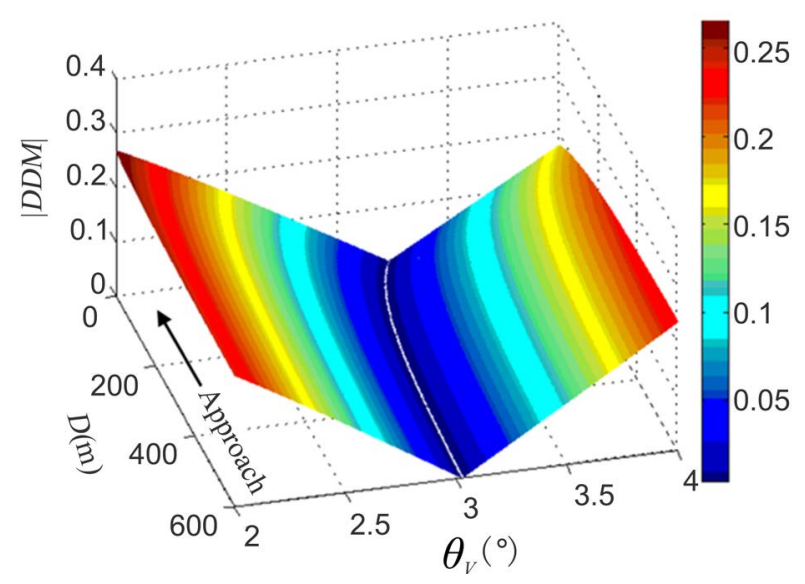

Figure 10. (Color Online) 3D pattern of ddm VS elevation angle from 0 to $600 \mathrm{~m}$. This is the horizontaldistance from the approach to runway entrance. The white curve indicate the zero point of $\mathrm{ddm}$, it can be found that approach to runway entrance, the elevation angle gradually rises. This phenomenon directly leads to the glide path upturned near the $\mathrm{TCH}$.

To research the NF condition further, the characteristic of ddm not only on glide path, but also in front of the NF are also studied. The angle $(\mathrm{ddm}=0)$ of two cases versus corresponding distance are showed in Figure 11, the inset gives the theoretical height versus the distance. For the case on glide path (case one), the red line in Figure 11, also the write line in Figure 10, indicates the angle is about $3.2^{\circ}$ at threshold, a litter higher than the glide path angle $\left(3^{\circ}\right)$ in far field. However the case of signal in front of the near field monitor (case two) is completely different. Firstly, the angle gradually becomes to lower when closes to the threshold. Secondly, there is a sharp decline within the distance of $1200 \mathrm{~m}$ (closes to the mark of NF: $1160 \mathrm{~m}$ ), declines to $1.8^{\circ}$ at threshold. This variation is 6 times $\left(\Delta \theta=1.2^{\circ}\right)$ than that on the glide path. Compare to the ddm distribution at near field monitor $(\sim 57 \mathrm{~m})$ position, the ddm distribution in $0-600 \mathrm{~m}$ is better, as least there is not false glide path, and the signal in their respective dominant area is right. The reason why the ddm distribution in case one is better than case two contributes to the lateral distance and it's corresponding lateral offset. To large extant, this alignment offsets the phase error both in FF and NF. The original purpose of the siting deviated from the one side of the runway is avoid touching aircraft. Lucky, this deviated siting also could improve near field signal by lateral offset.

\subsection{Comparison with M-Array Glide Path Antenna}

Among the former three types of image glide path antenna, the most comparable one is M-Array with three antennas as well as capture effect. Introduction below discusses the characteristic of offset, ddm distribution in NF, anti-interference capability, environment needs.

\subsubsection{Extent of Offset (Efficiency of Flight Check)}

After the glide path iron tower has been erected, it is not easy to move the position 


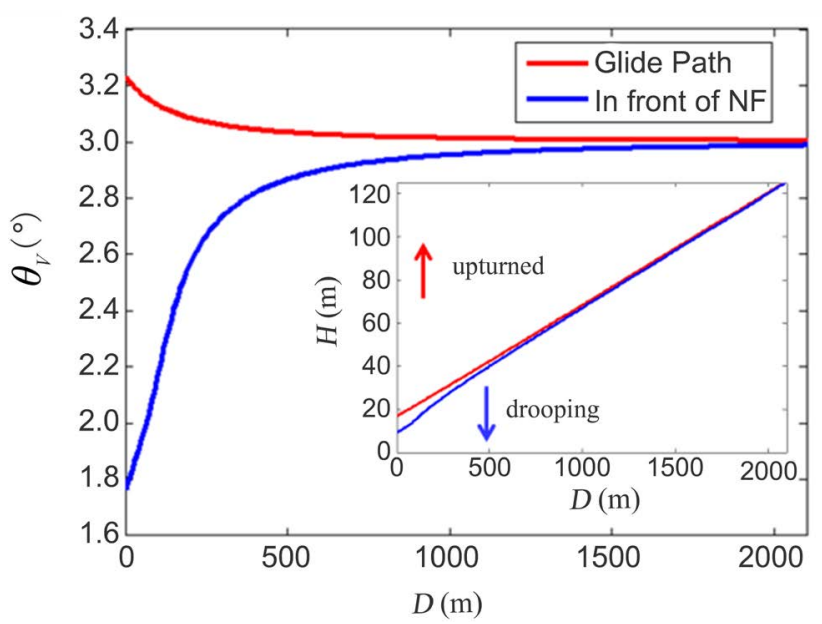

Figure 11. (Color Online) Distance to runway entrance dependence of elevation angle of $\mathrm{ddm}=0$. The inset indicates the height corresponding to the glide path line.

of each antenna, especially the old one which has been used for years. During the flight check, sometimes the position of the antenna should be aligned, such as mending glide angle by align the height of the middle antenna, optimizing the structure of III zone by adjusting offset, and improving TCH by twisting the antenna and so on. It is essential to change to the antenna position as soon as possible. In other words, the less extent, the quicker finished. The extent of several offsets of two types is listed in Table 3.

Theoretical calculation demonstrates that it is more easily to improving $\mathrm{TCH}$ for S-type than M-type by rotating the upper antenna. Obviously the S-type has the less changing extent. The parameter $h_{3}$ is the symbol of the size of the antenna system, the larger it is, the more expensive and inconvenient to maintain. The parameter $h_{3}-h_{1}$ represents the working range to maintain or repairmen. For example, if the transmitter cable is damaged, the ADU should be calibrated again after replaced the new cable, and operator would connect the cable up and down in this working region, the lager this region the poorer efficiency. And so do lateral offset adjustment.

\subsubsection{Near Field Signal}

The ddm distribution for near field monitor has been listed in Figure 9. Although the poor symmetry of the curve, it has both upper half width (ddm = $-0.0875)$ and lower half width ( $\mathrm{ddm}=0.0875)$. By contrast M-type just has the lower half width. And the linearity of S-type around the $\theta$ is more excellent than that of M-type.

For the monitor antenna, S-type is lower and has a shorter distance to glide path tower. This means the lower cost for manufacture and convenient to adjust monitor to the position of $\mathrm{ddm}=0$.

\subsubsection{Anti Interference Capability}

The beam bend potential (BBP) represents the anti-interference ability, the less bbp value the better it works. S-Array has the lowest radiation in lower angles to 
Table 3. Physical parameters of two types of antenna.

\begin{tabular}{ccccccc}
\hline Type & $h_{3}$ & $h_{3}-h_{1}$ & off $_{\mathrm{d}}$ & off $_{\mathrm{u}}$ & stf $_{\mathrm{d}}$ & stf $_{\mathrm{m}}$ \\
\hline M-array & 14.32 & 9.55 & 0.285 & -0.475 & 0.050 & 0.025 \\
S-array & 12.89 & 6.69 & 0.219 & -0.312 & 0.035 & 0.017 \\
$\Delta M$ & 0.99 & 0.66 & 0.038 & -0.064 & 0.034 & 0.017 \\
$\Delta S$ & 0.89 & 0.47 & 0.029 & -0.041 & 0.024 & 0.012 \\
\hline
\end{tabular}

Note: The case of $\theta=3^{\circ}, f=333.35 \mathrm{MHz}, F L S=0.3$ and $D=120 \mathrm{~m} . \Delta M$ means the variation from $F L S=0.3$ to $F L S=0.1$, the other parameters reserved. All the units of the data above are meter.

Table 4. BBP and CSB Ratio Comparison of four types.

\begin{tabular}{ccccc}
\hline Type & BBP $\left(1^{\circ}\right)$ & BBP $(\mu \mathrm{A})$ & CSB $\left(1^{\circ} / \mathrm{Max}\right)$ & Frequency \\
\hline Null Ref & $20.2 \%$ & 173 & $50 \%$ & Single \\
SB Ref & $14.8 \%$ & 127 & $26 \%$ & Single \\
M-Array & $2.7 \%$ & 23 & $5.2 \%$ & Double \\
S-Array & $0.04 \%$ & 0.34 & $0.5 \%$ & Double \\
\hline
\end{tabular}

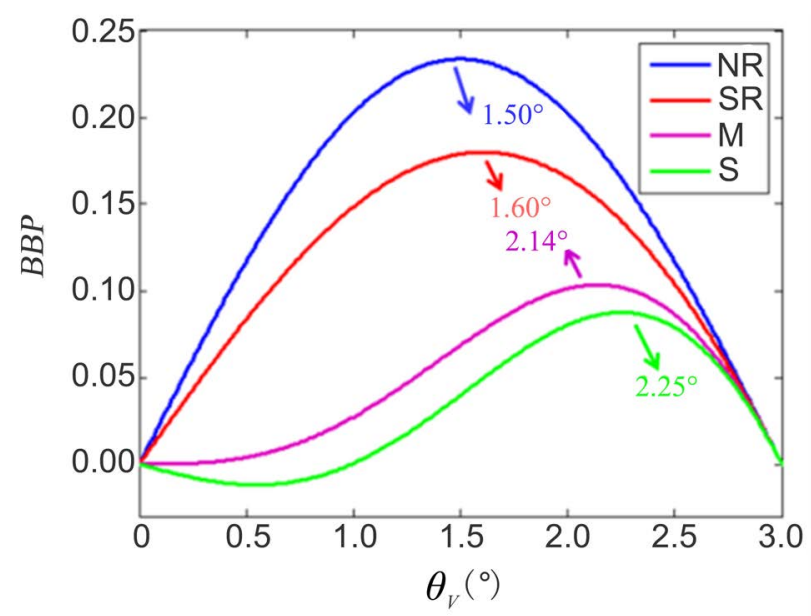

Figure 12. (Color Online) BBP pattern for four types of glide path antenna. The BBP value of null reference type is the maximum among four types, second one is sideband reference type, third one is M-type, while S-type is the minimum. The interesting phenomenon is the maximum value one (NR) corresponds the lowest angle $\left(1.5^{\circ}\right)$ from 0 to $3^{\circ}$, and the angle increasing with the bbp value decreasing, consequentially the S-type owns the highest angle $\left(2.25^{\circ}\right)$ with the lowest max value $(b b p \approx 0.09)$.

reduce illumination of terrain objects in order to reduce bends. Strictly speaking, the radiation of M-type is a little lower than S-type below $0.7^{\circ}$, because the S-type appears negative value, but after that, it owns the lower radiation up to $3^{\circ}$. As seen in Table 4, the S-type has the lowest bbp value at $1^{\circ}$, far less than the other three. This illustrates the good anti-interference ability for S-Array. Meanwhile the coverage parameter, CSB, chosen the value at $1^{\circ}$ divided by its maximum within $6^{\circ}$, shows that the S-type also has the minimum value. This confirms the satisfactory performance of low angle obstacle depressed (Figure 12). 


\section{Summary}

A new kind of image type glide path antenna was presented. To depress low angle radiation, the capture effect was also designed. And all of upper, middle, lower antennas radiated CSB and SBO signals. The calculation showed the CSB signal is $-41 \mathrm{~dB}$ at $1^{\circ}$ elevation angle comparative to the maximum value of CSB. DDM provided good linear variation around glide path angle.

To achieve a less length of reflection plane, the height of the system was reduced, which had only $90 \%$ of the M-array size, and needed $10 \%$ less length of reflection plane. Meanwhile, it achieved a shorter distance of NF, approximately $57 \mathrm{~m}$. This could save the cost of facility installation and maintenance. The shortened antennas gap results less size of offset, which could improve work efficiency in flight check adjusting.

\section{Conflicts of Interest}

The author declares no conflicts of interest regarding the publication of this paper.

\section{References}

[1] Watts Jr., C.B. (2003) The Instrument Landing System: Replace It, or Repair It? The Journal of Navigation, 56, 411-427.

[2] Normarc 7000B ILS (2013) Training Manual 24036-042.

[3] Normarc 3543 Null Reference Glide Path Antenna System Instruction Manual (2007) 21454-042, Park Air Systems.

[4] Normarc 3544 Sideband Reference Glide Path Antenna System Instruction Manual (2007) 21455-032, Park Air Systems.

[5] Normarc 3545 M-Array Glide Path Antenna System Instruction Manual (2007) 21456-045, Park Air Systems.

[6] Siting Criteria For Instrument Landing Systems (2004) 6750.16D. U.S. Department of Transportation Federal Aviation Administration.

[7] Annex 10 (2006) Aeronautical Telecommunications. Vol. I, 3.1.5.3.1, International Civil Aviation Organization. 